\title{
Determination of seminal plasma malondialdehyde by high-per- formance liquid chromatography in smokers and non-smokers
}

\author{
Stramova X, Kandar R \\ Department of Biological and Biochemical Sciences, Faculty of Chemical Technology, University of Pardubice, \\ Pardubice, Czech Republic. roman.kandar@upce.cz
}

\begin{abstract}
Objectives: The aim of this study was to establish and validate a high-performance liquid chromatography method for the determination of malondialdehyde in seminal plasma in smokers and non-smokers and to find possible differences between the two groups.

Background: Malondialdehyde is used as a diagnostic marker of lipid peroxidation and indicator of oxidative stress. Smoking is suspected to be responsible for an increase in its level. Malondialdehyde has been thought to have cytotoxic and damaging effects.

Methods: Semen samples were obtained from male partners of couples requesting a fertility evaluation. Malondialdehyde was derivatized with 2-thiobarbituric acid. The malondialdehyde-2-thiobarbituric acid complex was determined by liquid chromatography with fluorescence detection. The mobile phase consisted of $20 \%$ ethanol in $25-\mathrm{mmol} / \mathrm{L}$ potassium dihydrogenphosphate $(\mathrm{v} / \mathrm{v}), \mathrm{pH} 6.00 \pm 0.05$.

Results: Analytical performance was satisfactory. Malondialdehyde levels were as follows: $1.50 \pm 0.55 \mu \mathrm{mol} / \mathrm{L}$ in all patients, $1.40 \pm 0.57 \mu \mathrm{mol} / \mathrm{L}$ in smokers, and $1.50 \pm 0.53 \mu \mathrm{mol} / \mathrm{L}$ in non-smokers.

Conclusion: The method presented here is sensitive and accurate for seminal plasma malondialdehyde determination. Our results showed a relationship between sperm motility and the malondialdehyde level in all patients and non-smokers. Malondialdehyde may induce poor sperm functionality and negatively affect the fertilization processes (Tab. 1, Fig. 1, Ref. 23). Text in PDF www.elis.sk. Key words: malondialdehyde, semen, liquid chromatography, infertility.
\end{abstract}

\begin{abstract}
Abbreviations: ACN - acetonitrile; BMI - body mass index; DNPH - 2,4-dinitrophenylhydrazine; EDTA - ethylenediaminetetraacetic acid; GC - gas chromatography; HPLC - high-performance liquid chromatography; IQR - interquartile range; MDA - malondialdehyde; PUFA - polyunsaturated fatty acid; TBARS - thiobarbituric acid reactive substances.
\end{abstract}

Male infertility is becoming a very common problem in young men nowadays. The important pathological mechanisms leading to male infertility include sperm membrane damage by lipid peroxidation. The human sperm membrane is particularly susceptible to oxidative damage due to the high content of polyunsaturated fatty acids (PUFA) (1). The origin of lipid peroxides and lipid peroxidation metabolites is a cascade process. The initial products of PUFA oxidation are peroxyl radicals (2). These toxic products damage the process of spermatogenesis; thereby reducing the number of sperm cells and sperm motility, causing an inability

Department of Biological and Biochemical Sciences, Faculty of Chemical Technology, University of Pardubice, Pardubice, Czech Republic

Address for correspondence: R. Kandar, PhD, Department of Biological and Biochemical Sciences, Faculty of Chemical Technology, University of Pardubice, Studentska 573, CZ-532 10 Pardubice, Czech Republic. Phone: +420.466037714 , Fax: +420.466037068

Acknowledgements: This work was supported by a grant SGFChT07/2013. The authors thank the staff and patients of Sanus, Centre of Assisted Reproduction in Pardubice, for the seminal plasma samples. to undergo the acrosome reaction and fertilize (3). The degree of lipid peroxidation in seminal plasma can be determined in several ways. Malondialdehyde (MDA) is one of the final products of lipid peroxidation in seminal plasma (4). It is a highly reactive and genotoxic agent. MDA is able to react with DNA and proteins, thereby affecting physiological mechanisms in the body (5). It is therefore necessary to determine lipid peroxidation in human seminal plasma for the study of male infertility. Smoking, a potential free radical source, is thought to be one of the risk factors raising MDA level and may be responsible for sperm immobility and inability to fertilize.

MDA is most frequently determined as a thiobarbituric acid reactive substances (TBARS) after its reaction with TBA. MDA (TBA) $)_{2}$ absorbance is measured spectrophotometrically at 532 $\mathrm{nm}$. This method is simple and inexpensive, but TBA also reacts with many other compounds (e.g. amino acids, carbohydrates, etc.) (6). Separation methods have been developed, such as highperformance liquid chromatography (HPLC), gas chromatography (GC) and capillary electrophoresis. HPLC methods use different types of detectors, such as UV/Vis $(3,7)$, fluorescence (8), electrochemical $(9,10)$ and mass spectrometry detectors. Alternatively, derivatization with 2,4-dinitrophenylhydrazine (DNPH) (6) or diethylbarbituric acid (11) has been found to enable a specific estimation of MDA. Derivatization of MDA with pentafluorophenylhydrazine (6) or 2,4,6-trichlorohydrazine (12) enables the simultaneous determination of several aldehydes. 
The aim of this study was to establish and validate a HPLC method with fluorescence detection for the determination of MDA in the seminal plasma of patients from the Sanus Center of Assisted Reproduction, Pardubice and to compare MDA levels to spermiogram parameters in smokers and non-smokers.

\section{Materials and methods}

\section{Subjects and samples}

In this cross-sectional study conducted from September 2011 to May 2012 at the Sanus Center of Assisted Reproduction (Pardubice, Czech Republic), 50 male partners of couples requesting a fertility evaluation were selected to participate in the study. A couple is considered infertile if conception has not occurred after a year of regular unprotected sexual intercourse. About $15 \%$ of couples in the Czech Republic suffer from infertility. At present, men are the cause of about $50 \%$ of the cases of the couples' infertility and this number is constantly increasing.

The patients ( $31 \pm 5$ years of age) were divided into groups according to the completed questionnaire, into smokers $(n=18)$ and non-smokers $(\mathrm{n}=32)$. Smoking history was established through a questionnaire including questions on smoking activity. Based on the World Health Organization standards, if an individual had smoked 100 cigarettes in his lifetime, he was considered an active smoker. Such patients were placed in the smokers group in this study. In all cases, a written informed consent was obtained from all participants and the study was approved by the First Private Surgical Center Hradec Kralove Ethics Committee (2011). Sperm functionalities were examined according to the 2010 World Health Organization guidelines (13).

Semen samples were obtained by masturbation after $2-4$ days of sexual abstinence. After 35 minutes of liquefaction at room temperature, one part of the sample was used for estimating sperm functionalities and the second was centrifuged $(900 \mathrm{x}$ g, $5 \mathrm{~min}$, and room temperature) and the supernatant immediately frozen and stored at $-80^{\circ} \mathrm{C}$ until analysis. This procedure was conducted in the Sanus Center of Assisted Reproduction, Pardubice.

\section{Sample preparation}

Seminal plasma samples were collected into tubes containing butylated hydroxytoluene as an antioxidant. This approach was recommended in several studies $(3,6,14)$. Two hundred $\mu \mathrm{L}$ of $0.1 \%$ butylated hydroxytoluene in ethanol were pipetted into 1.5 $\mathrm{mL}$ polypropylene tubes. Ethanol was evaporated under a stream of pure nitrogen (Linde Gas a.s., Prague, Czech Republic). Tubes prepared in this manner were filled with $200 \mu \mathrm{L}$ of seminal plasma. $100 \mu \mathrm{L}$ of $0.1 \%$ EDTA solution and $50 \mu \mathrm{L}$ of $2-\mathrm{TBA}(28 \mathrm{mmol} / \mathrm{L})$ was added to $50 \mu \mathrm{L}$ of thawed seminal plasma sample. EDTA, as a chelating agent, inhibits lipid peroxidation in vitro (8). The mixture was vortexed for $60 \mathrm{~s}$ and incubated for $1 \mathrm{~h}$ at $100{ }^{\circ} \mathrm{C}$. After cooling, $1 \mathrm{~mL}$ of cold n-butanol was added; the mixture was vortexed for $10 \mathrm{~min}$ and centrifuged $\left(4000 \mathrm{x} \mathrm{g}, 30 \mathrm{~min}\right.$, and $\left.25^{\circ} \mathrm{C}\right)$. Organic solvents reduce the interference of other substances (3). The upper butanol layer was transferred into $1.0-\mathrm{mL}$ vials. MDA standards were prepared in the same way as the samples.

\section{Chromatography method}

The chromatographic analysis of MDA after their derivatization with TBA to form a stable, highly fluorescent derivative was accomplished using an isocratic elution in a LiChroCART ${ }^{\circledR}$ 125-4 Purospher ${ }^{\circledR}$ STAR-RP-18e, $5 \mu \mathrm{m}$ analytical column fitted with a LiChroCART® 4-4 Purospher® STAR-RP-18e, $5 \mu \mathrm{m}$ guard column (Merck KgaA, Darmstadt, Germany) at $37^{\circ} \mathrm{C}$. Mobile phase A was a mixture of ethanol and $25-\mathrm{mmol} / \mathrm{L}$ potassium dihydrogenphosphate $(20: 80, \mathrm{v} / \mathrm{v}), \mathrm{pH} 6.0$. A detectable influence of interfering substances occurred after several injections. The column was rinsed with mobile phase $\mathrm{B}$ (a mixture of ethanol and $25-\mathrm{mmol} / \mathrm{L}$ potassium dihydrogenphosphate 50:50, v/v, pH 6.0) to improve the separation step. Although the analysis time was prolonged, tailing peaks were avoided. The flow rate was kept constant at $0.5 \mathrm{~mL} /$ min. Optimum response of the fluorescence derivative was observed when the excitation and emission wavelengths were set to 532 and $553 \mathrm{~nm}$, respectively. The amount of MDA was quantified from the corresponding peak area of the fluorescence derivative using the chromatography software Clarity. The concentration of MDA in the samples was determined from its calibration curve (8).

\section{Chemicals and reagents}

Potassium dihydrogenphosphate, potassium hydroxide, phosphoric acid, acetic acid, n-butanol, 2-thiobarbituric acid, 1,1,3,3-tetramethoxypropane (the source of MDA standard), EDTA, butylated hydroxytoluene were obtained from Sigma (St. Louis, MO, USA). Ethanol gradient grade for HPLC was obtained from Merck KGaA (Darmstadt, Germany). All other chemicals were of analytical grade.

\section{HPLC instrumentation}

Chromatographic analysis was performed with a liquid chromatograph (Shimadzu, Kyoto, Japan) equipped with LC-10ADvp solvent delivery system, a SIL-10ADvp cooled autosampler, a CTO-10ACvp column oven, a RF-10Axl fluorescence detector, and a SCL-10Avp system controller. Data were collected digitally using the chromatography software Clarity (DataApex Ltd., Prague, Czech Republic).

\section{Statistical analysis}

The data are presented as the median $\pm \mathrm{IQR}$ (interquartile range). Differences between the non-smokers and the smokers were analyzed using the Mann-Whitney Rank Sum Test. The correlation analysis was carried out using the Spearman Rank-Order Correlation, and regression analysis was carried out using the least squares method (SigmaStat for Windows, Version 3.5, Systat Software Inc., Point Richmond, CA, USA). All statistical tests were two-tailed with statistical significance assessed at $p$-values $<0.05$ with $95 \%$ confidence intervals.

\section{Results}

\section{HPLC analysis of malondialdehyde}

Standard solutions of MDA as well as pooled seminal plasma were used for studying the mobile phase composition, $\mathrm{pH}$ value 


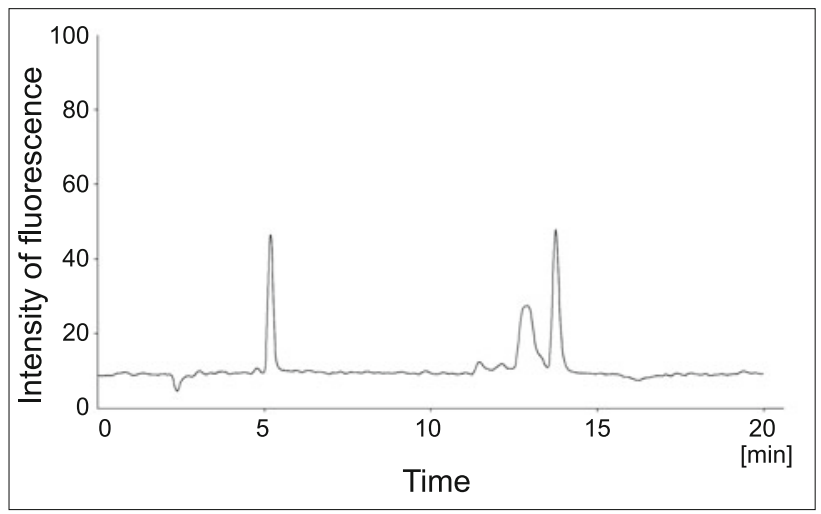

Fig. 1. An HPLC chromatogram of MDA in human seminal plasma $(0.89 \mu \mathrm{mol} / \mathrm{L})$. HPLC conditions: the mobile phase A was a mixture of $25-\mathrm{mmol} / \mathrm{L} \mathrm{KH}_{2} \mathrm{PO}_{4}$ and ethanol $(80: 20, \mathrm{v} / \mathrm{v}), \mathrm{pH} 6.0$, the mobile phase $B$ was a mixture of $25-\mathrm{mmol} / \mathrm{L} \mathrm{KH}_{2} \mathrm{PO}_{4}$ and ethanol $(50: 50) ; 0.00-8.00$ min.: mobile phase $A, 8.01-13.00$ min.: mobile phase $B, 13.01-20.00$ min.: mobile phase $A$, the flow rate was kept constant at $0.5 \mathrm{~mL} / \mathrm{min}$, optimum response of the fluorescence derivative was observed when the excitation and emission wavelengths were set to 532 and $553 \mathrm{~nm}$, respectively.

of the mobile phase, and column temperature. The best results were obtained for the conditions described in "Chromatography method". We prefer using an eco-friendly mobile phase, because organic solvents such as ACN and methanol are considered to be significant pollutants. According to our results, we can conclude that the method presented here is highly robust. An HPLC chromatogram of MDA in human seminal plasma is shown in Figure 1.

The calibration curve was linear over the whole tested range. The presented method is sensitive and accurate for seminal plasma MDA determination.

Determination of malondialdehyde in seminal plasma of male partners of couples requesting fertility evaluation

Fifty individuals attending the Sanus Center of Assisted Reproduction participated in this study. The study was held in two groups - smokers $(\mathrm{n}=18 ; 36.0 \%)$ and non-smokers $(\mathrm{n}=32$; $64.0 \%$ ). Age was recorded and BMI (body mass index) was calculated. Sperm parameters were determined at the Sanus Center of Assisted Reproduction, Pardubice according to the 2010 World Health Organization guidelines (13). Basic statistical analysis of the measured data is given in the Table 1 .

The Mann-Whitney Rank Sum Test was used to compare the parameters between smokers and non-smokers. There was no statistically significant difference between smokers and nonsmokers (Tab. 1).

MDA concentration, a marker of oxidative stress, was measured. The levels of MDA were $1.50 \pm 0.55 \mu \mathrm{mol} / \mathrm{L}$ in the group of all patients, $1.40 \pm 0.57 \mu \mathrm{mol} / \mathrm{L}$ in smokers, and $1.50 \pm 0.53$ $\mu \mathrm{mol} / \mathrm{L}$ in non-smokers.

The dependence of MDA level on selected spermiogram parameters, age, and BMI was determined. Correlations between the level of MDA and total sperm motility in all patients $(\mathrm{R}=-0.358$,

Tab. 1. Descriptive statistic of semen parameters, MDA concentration, age and BMI in smokers and non-smokers.

\begin{tabular}{|c|c|c|c|c|c|}
\hline $\begin{array}{l}\text { Parameter } \\
\text { (unit) }\end{array}$ & Group & $\mathrm{n}$ & Median & IQR & $* \mathrm{p}$ \\
\hline Age & All & 50 & 31.0 & 5.0 & \multirow{3}{*}{0.211} \\
\hline \multirow[t]{2}{*}{ (years) } & Smokers & 18 & 30.5 & 3.8 & \\
\hline & Non-smokers & 32 & 32.0 & 4.3 & \\
\hline \multirow{3}{*}{$\left(\mathrm{kg} / \mathrm{m}^{2}\right)$} & All & 50 & 26.2 & 4.1 & \multirow{3}{*}{0.461} \\
\hline & Smokers & 18 & 26.6 & 3.8 & \\
\hline & Non-smokers & 32 & 26.1 & 4.6 & \\
\hline \multirow{3}{*}{$\begin{array}{l}\text { MDA } \\
(\mu \mathrm{mol} / \mathrm{L})\end{array}$} & All & 50 & 1.50 & 0.55 & \multirow{3}{*}{0.832} \\
\hline & Smokers & 18 & 1.40 & 0.57 & \\
\hline & Non-smokers & 32 & 1.50 & 0.53 & \\
\hline \multirow{3}{*}{$\begin{array}{l}\text { Ejaculate volume } \\
(\mathrm{ml} / \mathrm{L})\end{array}$} & All & 50 & 3.5 & 2.0 & \multirow{3}{*}{0.776} \\
\hline & Smokers & 18 & 3.5 & 2.2 & \\
\hline & Non-smokers & 32 & 3.5 & 1.6 & \\
\hline \multirow{3}{*}{$\begin{array}{l}\text { Sperm cells amount } \\
\text { (mil) }\end{array}$} & All & 50 & 117.5 & 113.8 & \multirow{3}{*}{0.903} \\
\hline & Smokers & 18 & 111.3 & 78.3 & \\
\hline & Non-smokers & 32 & 122.5 & 151.8 & \\
\hline \multirow{3}{*}{$\begin{array}{l}\text { Sperm concentration } \\
(\mathrm{mil} / \mathrm{ml} / \mathrm{L})\end{array}$} & All & 50 & 35.5 & 40.8 & \multirow{3}{*}{0.903} \\
\hline & Smokers & 18 & 37.0 & 29.5 & \\
\hline & Non-smokers & 32 & 35.0 & 45.3 & \\
\hline \multirow{3}{*}{$\begin{array}{l}\text { Total motility } \\
(\%)\end{array}$} & All & 50 & 77.1 & 18.8 & \multirow{3}{*}{0.413} \\
\hline & Smokers & 18 & 77.8 & 15.5 & \\
\hline & Non-smokers & 32 & 76.2 & 21.1 & \\
\hline \multirow{3}{*}{$\begin{array}{l}\text { Progressive motility } \\
(\%)\end{array}$} & All & 50 & 61.0 & 21.4 & \multirow{3}{*}{0.872} \\
\hline & Smokers & 18 & 60.7 & 14.6 & \\
\hline & Non-smokers & 32 & 61.3 & 23.0 & \\
\hline \multirow{3}{*}{$\begin{array}{l}\text { Morphology } \\
(\%)\end{array}$} & All & 50 & 10.0 & 10.5 & \multirow{3}{*}{0.792} \\
\hline & Smokers & 18 & 10.5 & 7.3 & \\
\hline & Non-smokers & 32 & 10.0 & 11.5 & \\
\hline
\end{tabular}

* $\mathrm{p}<0.05$ Mann - Whitney Rank Sum test; IQR - interquartile range (difference between 75th and 25th percentiles); BMI - body mass index 
$\mathrm{p}=0.013$ ), between the level of MDA and progressive motility $(\mathrm{R}=-0.465, \mathrm{p}=0.001)$ in non-smokers and between MDA level and total sperm motility in non-smokers $(\mathrm{R}=-0.382, \mathrm{p}=0.037)$ were found.

\section{Discussion}

Spermatogenesis is affected by many factors. One of the causes of male infertility is oxidative stress. Frequent smoking contributes to a reduction in antioxidant levels in seminal plasma and the deterioration of spermiogram parameters. Male infertility is affected by many external factors (e.g. environmental, physiological, and genetic factors) (15). Cigarette smoke contains known mutagenic and carcinogenic substances $(16,17)$. The metabolites of cigarette smoke are thought to increase MDA level and lipid peroxidation processes. Therefore, sperm cells membranes are diminished and spermatozoa are not able to fertilize because of immobility. Recent studies have shown the active transfer of several components of cigarette smoke through the blood-testis barrier (16). As a result, it is important to study the factors affecting male fertility, especially MDA.

Many studies have proposed a correlation between smoking and changes in ejaculate quality but conflicting results have been obtained. Important variables affected sperm concentration, morphology and motility (16). It has been shown that the MDA concentration in seminal plasma is significantly related to the number of immobile sperm cells (18). A negative correlation of seminal plasma MDA with sperm motility and concentration has been demonstrated (19). Also, a negative correlation of MDA level with sperm morphology was confirmed (20). Another study claims that the MDA concentration in seminal plasma does not correlate with sperm concentration, motility, or the number of immobile sperm cells (21).

The negative correlation between MDA levels and sperm motility can be explained. Sperm membranes contain a large amount of PUFA; therefore they are highly susceptible to lipid peroxidation. An increased amount of MDA signifies increased lipid peroxidation. This may cause membrane damage and reduce membrane integrity and sperm motility. This conclusion was supported by several studies $(1,16,18,19,22,23)$.

Lipid peroxidation has a damaging effect on sperm quality. An increased MDA level in seminal plasma may correlate with or even be responsible for the pathophysiology of male infertility.

High-performance liquid chromatography with fluorescence detection for the determination of MDA in seminal plasma was optimized. The analytical parameters of the method were satisfactory and this method is appropriate for determination of MDA in seminal plasma.

MDA levels were not significantly different between smokers and non-smokers. Neither were there any significant differences found in individual parameters of the spermiogram. The only statistically significant correlations were found between the MDA level and total sperm motility in all patients, between MDA level and progressive motility in non-smokers and between MDA level and total sperm motility in non-smokers.
The measurement of MDA could be useful diagnostic tool for oxidative stress estimation. However, MDA originates from smoking, may induce poor sperm motility and also negatively affect fertilization processes. Also, smokers should quit smoking for the sense of responsibility for their future generation and their own health.

\section{References}

1. Tavilani H, Doosti M, Saeidi H. Malondialdehyde levels in sperm and seminal plasma of asthenozoospermic and its relationship with semen parameters. Clin Chim Acta 2005; 356: 199-203.

2. Marnett L.J. Lipid peroxidation-DNA damage by malondialdehyde. Mutat Res 1999; 424: 83-95.

3. Li K, Shang X, Chen Y. High-performance liquid chromatographic detection of lipid peroxidation in human seminal plasma and its application to male infertility. Clin Chim Acta 2004; 346: 199-203.

4. Dermani KF, Colagar HA. Effects of cigarette smoking on seminal TAC and MDA levels in fertile and infertile men. Int J Fertil Steril 2012; 6: 133 .

5. Del Rio D, Stewart AJ, Pellegrini N. A review of recent studies on malondialdehyde as toxic molecule and biological marker of oxidative stress. Nutr Metab Cardiovasc Dis 2005; 15: 316-328.

6. Mateos R, Lecumberri E, Ramos S, Goya L, Bravo L. Determination of malondialdehyde (MDA) by high-performance liquid chromatography in serum and liver as a biomarker for oxidative stress. Application to a rat model for hypercholesterolemia and evaluation of the effect of diets rich in phenolic antioxidants from fruits. J Chromatogr B 2005; 827: 76-82.

7. Khoschsorur GA, Winklhofer-Roob BM, Rabl H, Auer T, Peng Z, Schaur RJ. Evaluation of a sensitive HPLC method for the determination of malondialdehyde, and application of the method to different biological materials. Chromatographia 2000; 52: 181-184.

8. Kand’ár R, Mužáková V, Čegan A. Highly specific, simple and rapid method for the determination of malondialdehyde in blood using highperformance liquid chromatography. Clin Chem Lab Med 2002; 40: 1032-1035.

9. Guichardant M, Valette-Talbi L, Cavadini C, Crozier G, Berger M. Malondialdehyde measurement in urine. J Chromatogr B 1994; 655: 112-116.

10. Stalikas CD, Konidari CN. Analysis of malondialdehyde in biological matrices by capillary gas chromatography with electron-capture detection and mass spectrometry. Anal Biochem 2001; 290: 108-115.

11. Wilson DW, Metz HN, Graver ML, Rao PS. Direct method for quantification of free malondialdehyde with high-performance capillary electrophoresis in biological samples. Clin Chem 1997; 10: 1982-1984.

12. Claeson K, Thorsen G, Karlberg B. Methyl malondialdehyde as an internal standard for the determination of malondialdehyde. J Chromatogr B 2001; 751: 315-323.

13. World Health Organization, WHO laboratory manual for the examination and processing of human semen 2010 (pp.7-173). Geneva, Switzerland.

14. Verbunt RJ, Egas JM, Van der Laarse A. Risk of overestimation of free malondialdehyde in perfused rat hearts due to homogenization artifacts. Cardiovasc Res 1996; 31: 603-606. 
$20-24$

15. Safdar M, Azima S. Lifestyle factors in deteriorationg male reproductive health. Int J Fertil Steril 2010; 4 (1): 58.

16. Aryanpur M, Tarahomi M, Sharifi $\mathbf{H}$ et al. Comparison of spermatozoa quality in male smokers and nonsmokers of Iranian infertile couples. Int J Fertil Steril 2011; 5: 152-157.

17. Mostafa T. Cigarette smoking and male infertility. J Adv Res 2010; 1: $179-186$.

18. Kobayashi T, Miyazaki T, Natori M, Nozawa S. Protective role of superoxide dismutase in human sperm motility: superoxide dismutase activity and lipid peroxide in human seminal plasma and spermatozoa. Hum Reprod 1991; 6: 987-991.

19. Hsieh Y, Chang C, Lin C. Seminal malondialdehyde concentration but not glutathione peroxidase activity is negatively correlated with concentration and motility. Int J Biol Sci 2006; 2: 23-29.
20. Chari MG, Colagar AH. Seminal plasma lipid peroxidation, total antioxidant capacity, and cigarette smoking in asthenoteratospermic men. J Men Health 2011; 8: 43-49.

21. Suleiman SA, Ali ME, Zaki ZM, El-Malik EM, Nasr MA.Lipid peroxidation and human sperm motility: protective role of vitamin E. J Androl 1996; 17: 530-537.

22. Franczek M, Szkutnik D, Sanocka D, Kurpisz M. Peroxidation components of sperm lipid membranes in male infertility. Ginekol Pol 2001; 72: 73-79.

23. Nabil H, Moemen LA, Abu Elela MH. Studying the levels of malondialdehyde and antioxidant parameters in normal and abnormal human seminal plasma. Aust J Bas Appl Sci 2008; 2: 773-778.

Received Accepted September 20, 2014. 